\title{
ELASTIC HYDRODYNAMIC LUBRICATION OF TWO-STEP SINE MOVABLE TOOTH DRIVE
}

\section{LIZHONG XU and PEINI YAO}

\author{
Yanshan University \\ Mechanical Engineering Institute \\ Qinhuangdao, 066004 \\ P. R. China \\ e-mail: xlz@ysu.edu.cn
}

\begin{abstract}
Radial size of two-step sinusoidal movable tooth drive is small, especially suitable for robot arm and other technical fields requiring compacting. However, as the size of the drive decreases, the lubrication state of the meshing pair becomes worse and should be improved. In this paper, the minimum oil film thickness of the elastic hydrodynamic lubrication for the drive system is investigated by determining the suction velocity of the moving pair, the radius of curvature and the load per unit length. The influence of the drive parameters on the minimum oil film thickness is analyzed. Results show that the minimum oil film thickness of the drive system can be increased by increasing the eccentric distance of the eccentric wheel, center distance between the movable tooth and the eccentric wheel, and radius of the movable tooth. The good lubrication state can be achieved by using proper tooth surface processing technology.
\end{abstract}

2010 Mathematics Subject Classification: 00A69.

Keywords and phrases: minimum oil film thickness, elastic hydrodynamic lubrication, twostep sine movable tooth drive.

Received March 21, 2019

(C) 2019 Scientific Advances Publishers 


\section{Introduction}

Mechanical drive technology is an important symbol of the development of mechanical equipment. The movable tooth drive has a novel structure, high speed ratio and high efficiency. It can be widely used in aerospace, petroleum and precision instruments and other technical fields [1, 2].

In 80-90's of the 20th century, reducer without teeth and novel differential gear reducer system were proposed [3, 4]. Since the beginning of the 21st century, the research on movable tooth drives has been increasing. In 2009-2010, Terada and Imase [5, 6] proposed a twostep ball tooth drive and calculated its profile. In 2013, Han [7] designed the electric roller based on movable tooth drive, which extended the service life of the electric roller. In 2014, Kong [8] developed a precise cycloidal movable tooth drive reducer suitable for heavy duty industrial robot. In 2015, Wang and Chang [9] investigated tooth profile of swing movable teeth transmission and analyzed meshing curve of the transmission. In 2015-2016, Liang and $\mathrm{Xu}$ [10, 11] put forward electromagnetic harmonic movable tooth drive system. In 2003-2017, Liang et al. [12, 13] established the dynamic model of swing movable tooth drive and completed its modal analysis and investigation of the thermo-elastic coupling deformation of tooth surface. In 2017, Li et al. [14] investigated chaotic vibration of drive system for a movable tooth piezoelectric motor. A new two-stage sinusoidal movable tooth transmission was presented in 2017. Its remarkable advantages are large speed ratio, small volume, and especially suitable for compact structure situations such as robot and oil drilling [15]. Forces and stress in the novel two-step movable tooth drive were analyzed [16]. However, the reduction of drive size also reduces the area of heat dissipation, resulting in high temperature rise when lubrication is poor. Therefore, it is necessary to study the elasto-hydrodynamic lubrication of two-stage sinusoidal movable tooth drive. 
In this paper, the basic principle of a two-stage sinusoidal movable tooth drive is introduced. Equations of the suction velocity of the moving pair, the radius of curvature and the load per unit length for the drive are deduced. Based on it, the equation of the minimum oil film thickness of the elastic hydrodynamic lubrication for the drive system is determined. Using the equation, the minimum oil film thickness of the elastic hydrodynamic lubrication for the drives system is investigated and the effects of the drive parameters on the minimum oil film thickness are analyzed. The research results can be used to design lubrication state of the two-stage sinusoidal movable tooth drive.

\section{Basic Principle of Two-Stage Sinusoidal Movable Tooth Drive}

The two stage sinusoidal movable tooth drive combines the space movable tooth drive with the plane movable tooth drive, and reduces the axial dimension while realizing a large speed ratio. Structure of twostage sinusoidal movable teeth drive, as shown in Figure 1, consists of a input shaft, a guide frame, a shell, a movable tooth carrier, a central wheel and two teams of the movable teeth. The motor inputs the power to the input shaft, which rotates at a uniform speed, moves the movable teeth under the push of a single cycle closed slot. This is constrained by the multi-period closed slot on the shell, and moves around the common axis of the input shaft and the shell at equal speed. The eccentric wheel on the guide frame serves as the output of the first stage and the input of the second stage, which effectively connects the two stages of drives. When the eccentric wheel rotates at a uniform speed, the single cycle closed slot on the eccentric wheel drives the movable teeth to move along the multi-period closed slot on the central wheel, while the movable tooth inversely pushes the movable tooth carrier to rotate at a uniform speed, thus completing the rotational speed transformation. 


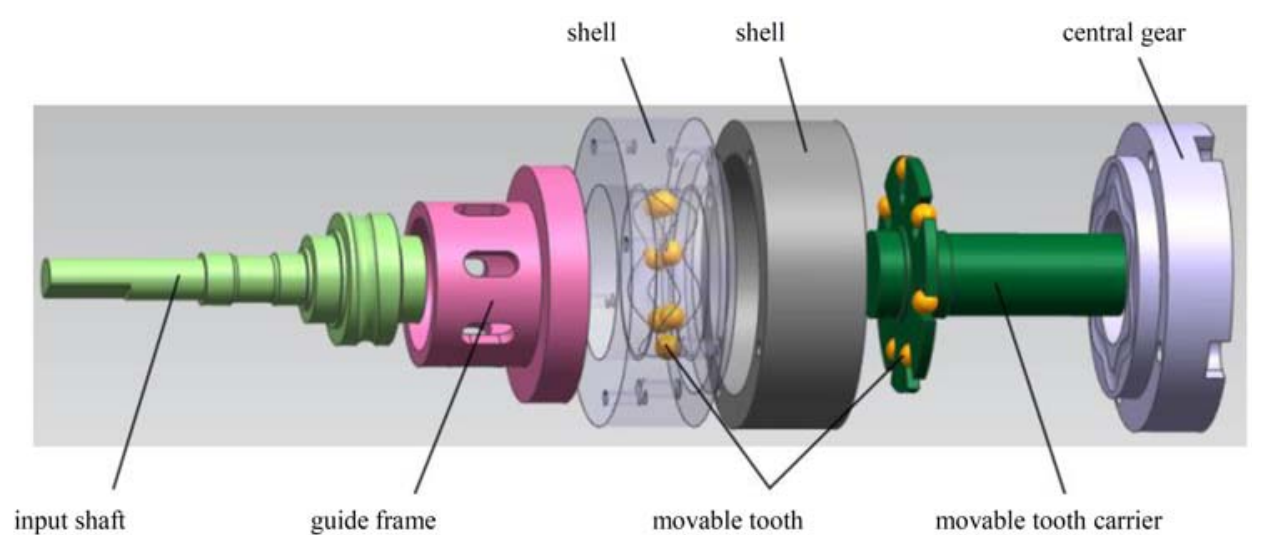

Figure 1. Two-step sine movable tooth drive.

\section{Minimum Oil Film Thickness in EHL}

The meshing process between the movable teeth and the inner or outer sinusoidal raceways is very complicated in the two-step sinusoidal movable tooth drive, because the direction and values of the entrainment velocity at different meshing points change. So, the oil film thickness is very uneven. In addition, the oil film thickness will become smaller when the viscosity of lubricating oil decreases due to friction temperature rise. The two-step sinusoidal movable tooth drive is a reducer system. The first stage is a space movable tooth drive, and the second stage is a plane movable tooth drive. The moving pair of the second stage drive has a smaller entrainment speed and a thinner oil film. That is, the lubrication state of the second stage movable tooth drive determines the whole lubrication state of the drive system. The important sign of the lubricating state of the lubricating parts is the oil film thickness. Therefore, this paper only studies the minimum oil film thickness in the second stage drive.

For gear and rolling bearing, point or line contact occurs so contact pressure is quite high. It will increase the viscosity of lubricant, and high pressure will cause elastic deformation of the contact elements. This lubrication problem, which takes into account the influence of elastic 
deformation and pressure-viscosity variation on hydrodynamic lubrication, is called elasto-hydrodynamic lubrication [17-19]. The minimum oil film thickness is calculated by Dowson-Higginson formula [19]:

$$
H_{\min }=2.65 \frac{\alpha^{0.54}\left(\eta_{0} \cdot U\right)^{0.7} R_{S}^{0.43}}{E^{\prime 0.03} \cdot W^{0.13}}
$$

where $H_{\text {min }}$ is the minimum oil film thickness; $\alpha$ is pressure viscosity coefficient; $\eta_{0}$ is dynamic viscosity of lubricant under normal pressure; $U$ is entrainment velocity; $E^{\prime}$ is effective elastic modulus; $W$ is load per unit length; and $R_{S}$ is effective curvature radius.

In the second stage of sinusoidal movable tooth drive, there are three basic parts: the guide frame (eccentric wheel, $H$ ), the movable tooth carrier $(G)$, and the center wheel $(K)$.

Their angular velocities are $\omega_{2}, \omega_{3}$, and $\omega_{H}$, respectively (counterclockwise), in which the center wheel is fixed, so $\omega_{H}=0$. An additional angular velocity $\left(-\omega_{3}\right)$ is now added to the whole movable tooth drive. According to the principle of relative motion, there is no influence on the relative motion between any two components in the movable tooth drive. In this case, the movement state of each component becomes: the eccentric wheel rotates counterclockwise at the angular velocity of $\left(\omega_{2}-\omega_{3}\right)$, the movable tooth carrier is fixed, and the center wheel rotates clockwise at the angular velocity of $\omega_{3}$.

The speed ratio of the second stage drive is $i_{2}=\frac{Z_{2}+Z_{4}}{Z_{2}}=\frac{\omega_{2}}{\omega_{m}} \cdot \frac{S_{r}}{r}$, here $Z_{2}$ is period number of tooth slot on eccentric wheel, $Z_{4}$ is period number of the tooth slot on central wheel. Then, the angular velocity of the movable tooth around its axis is

$$
\omega_{m}=\frac{\omega_{2} S_{c}}{i_{2} r},
$$


where $S_{c}$ is theoretical tooth profile of central wheel, $S_{c}=a \cos Z_{4} \varphi_{3}$ $+\sqrt{b^{2}-a^{2} \sin ^{2} Z_{4} \varphi_{3}}$

$\varphi_{3}$ is rotation angle of the movable tooth carrier; and $r$ is radius of the movable tooth.

The motion velocity of the movable tooth is

$$
V_{m}=\omega_{m} \cdot r
$$

The motion velocity of the eccentric wheel is

$$
V_{H}=\left(\omega_{2}-\omega_{3}\right) \cdot R_{P}
$$

where $R_{p}$ is radius of the eccentric wheel.

The motion velocity of the central wheel is

$$
V_{K}=-\omega_{3} \cdot S_{c}
$$

Thus, entrainment velocities between the movable tooth and eccentric wheel, movable tooth carrier, or central wheel are

$$
\left.\begin{array}{c}
U_{1}=\frac{1}{2}\left(V_{m}+V_{H}\right)=\frac{1}{2}\left[\frac{\omega_{2} S_{c}}{i_{2}}+\left(\omega_{2}-\omega_{3}\right) R_{P}\right] \\
U_{2}=\frac{1}{2} V_{m}=\frac{1}{2} \frac{\omega_{2} S_{c}}{i_{2}} \\
U_{3}=\frac{1}{2}\left(V_{m}+V_{K}\right)=\frac{1}{2}\left(\frac{\omega_{2} S_{c}}{i_{2}}+\omega_{3} S_{c}\right)
\end{array}\right\} .
$$

The curvature radius of a point on a surface is the radius of a close circle of that point. The movable tooth is a sphere, and the close circle of each point is itself, so its radius of curvature is its own radius.

The sinusoidal raceway with different periods is processed on the eccentric wheel and the central wheel. The section of the raceway is circular, so the curvature radius of the raceway is the raceway radius. The tooth surface of the movable tooth and the sinusoidal raceway are two conjugate surfaces, and the two conjugate surfaces can be considered 
as internal meshing. In summary, the effective radius of curvature can be obtained from the induced normal curvature. It is

$$
\left.\begin{array}{c}
R_{S}=\left|\frac{1}{k_{v}}\right| \\
k_{v}=k-k^{\prime}
\end{array}\right\},
$$

where $k$ is curvature of the movable tooth, $k=1 / r ; k^{\prime}$ is curvature of the tooth slot.

The track curve of sinusoidal raceway on eccentric wheel is as follows:

$$
\left\{\begin{array}{l}
x=r^{\prime} \cos t \\
y=r^{\prime} \sin t
\end{array}\right.
$$

From Equation (8), curvature $k^{\prime}$ of the tooth slot can be obtained

$$
k^{\prime}=\frac{|\dddot{x} \ddot{y}-\dot{y} \ddot{x}|}{\left(\dot{x}^{2}+\dot{y}^{2}\right)^{3 / 2}} .
$$

The load per unit length between the meshing pair is

$$
W_{n}=\frac{F_{n i}^{(2)}}{L},(n=1,2,3)
$$

where $F_{n i}^{(2)}$ is normal force applied to movable tooth and $L$ is length of the contact line

$$
L=r \sin \left(\frac{\pi}{2}-\gamma\right)
$$

where $\gamma=\arcsin \left(\frac{1}{\lambda} \sin Z_{4} \varphi_{3}\right) ; \lambda$ is the wave coefficient

Forces on the second stage movable tooth drive are shown in Figure 2. From Figure 2, the angle $\alpha$ can be calculated as

$$
\alpha=\frac{d y^{\prime}}{d x^{\prime}}=\arctan \left(-\frac{Z_{4} \sin Z_{4} \varphi_{3} \cos \varphi_{3}+\sqrt{\lambda^{2}-\sin ^{2} Z_{4} \varphi_{3}} \sin \varphi_{3}}{Z_{4} \sin Z_{4} \varphi_{3} \sin \varphi_{3}-\sqrt{\lambda^{2}-\sin ^{2} Z_{4} \varphi_{3}} \cos \varphi_{3}}\right) .
$$




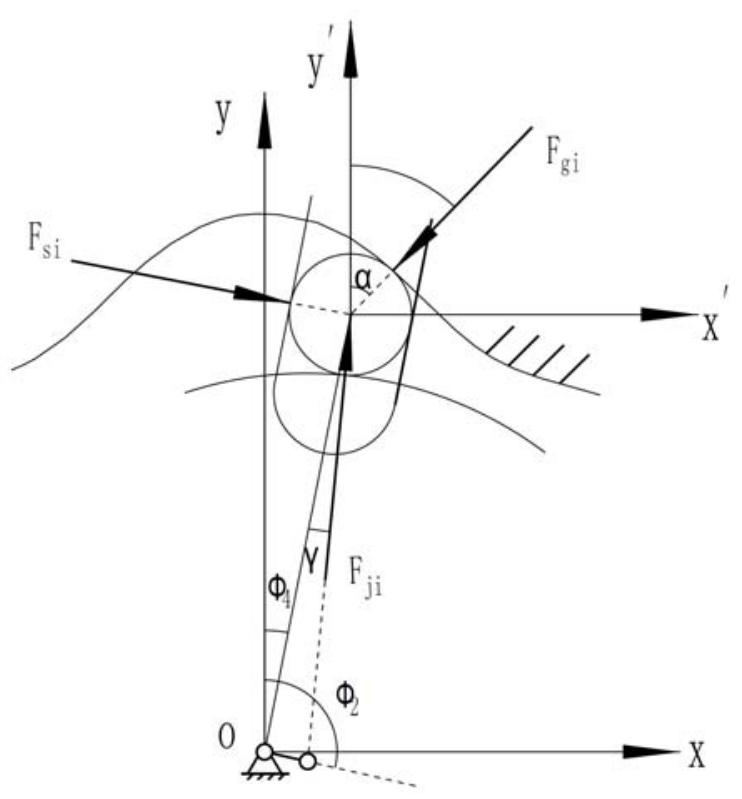

(a) forces on tooth in horizontal plane

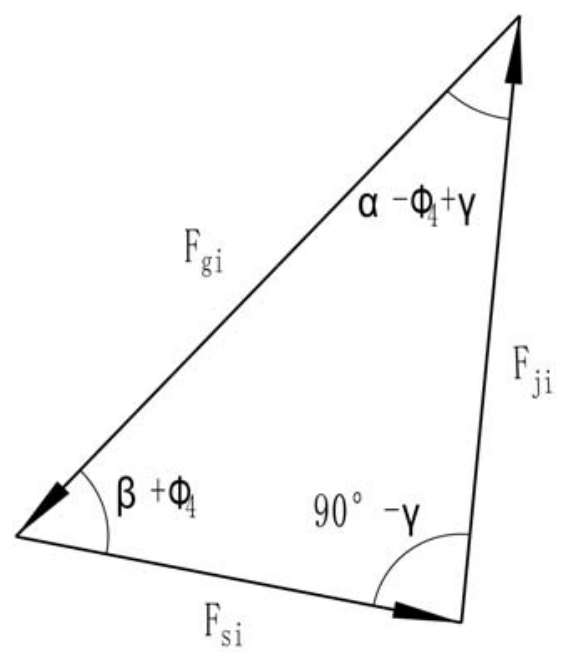

(b) relation of three forces 


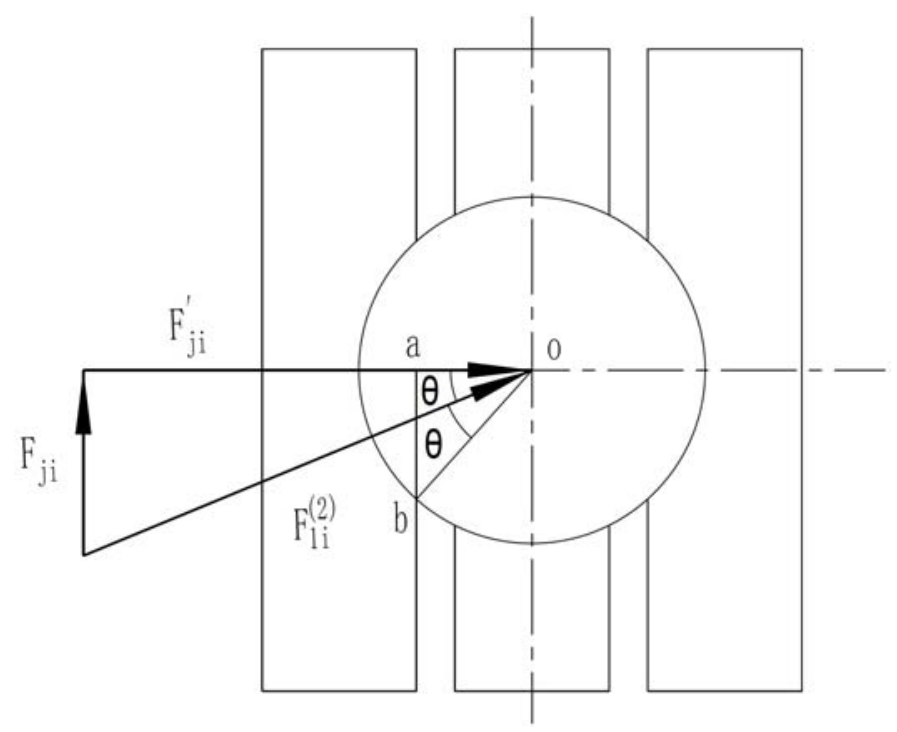

(c) forces on tooth in vertical plane

Figure 2. Forces in the second step drive.

Three forces are subjected to the movable tooth, which includes force from eccentric wheel $\left(F_{j i}\right)$, force from the movable tooth frame $\left(F_{s i}\right)$, and the force from central wheel $\left(F_{g i}\right)$. From Figure 2(b), it is known

$$
\frac{F_{s i}}{\sin \left(\alpha-\varphi_{3 i}+\gamma\right)}=\frac{F_{j i}}{\sin \left(\beta+\varphi_{3 i}\right)} .
$$

Substituting $\alpha$ and $\gamma$ into (13), yields

$$
F_{s i}=\left(\frac{i_{2}}{\lambda} \sin Z_{4} \varphi_{3 i}\right) \cdot F_{j i}
$$

In a same manner, it is given as well

$$
F_{g i}=\left(\frac{1}{\lambda} \sqrt{\left(Z_{4}^{2}-1\right) \sin ^{2} Z_{4} \varphi_{3 i}+\lambda^{2}}\right) \cdot F_{j i}
$$


Under the torque, the elastic contact deformation between the movable tooth and the eccentric wheel occurs. It changes in a sinusoidal function.

The relationship between the elastic deformation $\varepsilon_{i}$ at any position and the maximum elastic deformation $\varepsilon$ is:

$$
\varepsilon_{i}=\frac{\varepsilon}{\lambda}\left(\cos Z_{4} \varphi_{3 i}+\sqrt{\lambda^{2}-\sin ^{2} Z_{4} \varphi_{3 i}}\right) \sin Z_{4} \varphi_{3 i} .
$$

From Hooke law, the relationship between the force $F_{j i}$ at any position and the maximum force $F_{j}$ is

$$
F_{j i}=\frac{F_{j}}{\lambda}\left(\cos Z_{4} \varphi_{3 i}+\sqrt{\lambda^{2}-\sin ^{2} Z_{4} \varphi_{3 i}}\right) \sin Z_{4} \varphi_{3 i},
$$

where

$$
\begin{gathered}
F_{j}=\frac{\lambda^{2} \cdot T_{n 1}}{Z_{t}} ; \\
Z_{t}=\sum_{k=0}^{n} \sin ^{2} Z_{4} \varphi_{3 i}\left(\cos Z_{4} \varphi_{3 i}+\sqrt{\lambda^{2}-\sin ^{2} Z_{4} \varphi_{3 i}}\right) \\
\cdot\left[a\left(\cos Z_{4} \varphi_{3 i}+\sqrt{\lambda^{2}-\sin ^{2} Z_{4} \varphi_{3 i}}\right)-r\right] .
\end{gathered}
$$

Substituting Equation (17) into (14) and (15) yields

$$
\left.\begin{array}{rl}
F_{j i}=\frac{T_{n 1} \lambda}{Z_{t}}\left(\cos Z_{4} \varphi_{3 i}+\sqrt{\lambda^{2}-\sin ^{2} Z_{4} \varphi_{3 i}}\right) \sin Z_{4} \varphi_{3 i} \\
F_{s i}=\frac{T_{n 1} i_{2}}{Z_{t}}\left(\cos Z_{4} \varphi_{3 i}+\sqrt{\lambda^{2}-\sin ^{2} Z_{4} \varphi_{3 i}}\right) \sin ^{2} Z_{4} \varphi_{3 i} \\
F_{g i}=\frac{T_{n 1} \lambda}{Z_{t}}\left(\cos Z_{4} \varphi_{3 i}+\sqrt{\lambda^{2}-\sin ^{2} Z_{4} \varphi_{3 i}}\right) \\
\cdot \sin Z_{4} \varphi_{3 i} \cdot \sqrt{\left(Z_{3}^{2}-1\right) \sin ^{2} Z_{4} \varphi_{3 i}+\lambda^{2}}
\end{array}\right\} .
$$


Forces on tooth in vertical plane are shown in Figure 2(c). From Figure 2(c), it is given

$$
\sin \theta=\frac{1}{2}\left(1-\frac{o a}{o b}\right)
$$

Thus, the forces on the movable tooth can be obtained

$$
\left.\begin{array}{r}
F_{1 i}^{(2)}=\frac{T_{n 1} \lambda}{Z_{t} \sin \theta}\left(\cos Z_{4} \varphi_{3 i}+\sqrt{\lambda^{2}-\sin ^{2} Z_{4} \varphi_{3 i}}\right) \sin Z_{4} \varphi_{3 i} \\
F_{2 i}^{(2)}=\frac{T_{n 1} i_{2}}{Z_{t}}\left(\cos Z_{4} \varphi_{3 i}+\sqrt{\lambda^{2}-\sin ^{2} Z_{4} \varphi_{3 i}}\right) \sin ^{2} Z_{4} \varphi_{3 i} \\
F_{3 i}^{(2)}=\frac{T_{n 1} \lambda}{Z_{t} \sin \theta}\left(\cos Z_{4} \varphi_{3 i}+\sqrt{\lambda^{2}-\sin ^{2} Z_{4} \varphi_{3 i}}\right) \sin Z_{4} \varphi_{3 i} \\
\cdot \sqrt{\left(Z_{3}^{2}-1\right) \sin ^{2} Z_{4} \varphi_{3 i}+\lambda^{2}}
\end{array}\right\} .
$$

Substituting Equation (22) into (10), the load per unit length between the meshing pair can be given

$$
\left.\begin{array}{c}
W_{1}=\frac{T_{n 1} \lambda}{Z_{t} r \sin \theta \sin \left(\frac{\pi}{2}-\gamma\right)}\left(\cos Z_{4} \varphi_{3 i}+\sqrt{\lambda^{2}-\sin ^{2} Z_{4} \varphi_{3 i}}\right) \sin Z_{4} \varphi_{3 i} \\
W_{2}=\frac{T_{n 1} i_{2}}{Z_{t} r \sin \left(\frac{\pi}{2}-\gamma\right)}\left(\cos Z_{4} \varphi_{3 i}+\sqrt{\lambda^{2}-\sin ^{2} Z_{4} \varphi_{3 i}}\right) \sin ^{2} Z_{4} \varphi_{3 i} \\
W_{3}=\frac{T_{n 1} \lambda}{Z_{t} r \sin \theta \sin \left(\frac{\pi}{2}-\gamma\right)}\left(\cos Z_{4} \varphi_{3 i}+\sqrt{\lambda^{2}-\sin ^{2} Z_{4} \varphi_{3 i}}\right) \\
\cdot \sin Z_{4} \varphi_{3 i} \cdot \sqrt{\left(Z_{3}^{2}-1\right) \sin ^{2} Z_{4} \varphi_{3 i}+\lambda^{2}}
\end{array}\right\}
$$

where $W_{1}, W_{2}$, and $W_{3}$ are loads per unit length between the movable tooth and eccentric wheel, movable tooth frame, or central wheel, respectively. Substituting Equations (6), (7), and (23) into (1), the minimum oil film thickness between the movable tooth and eccentric wheel, movable tooth frame, or central wheel can be determined.

\section{Results and Discussion}

Using above equations, the minimum oil film thickness at different positions of the drive system is simulated. Table 1 gives parameter values example drive system. The torque is $140 \mathrm{~N} \cdot \mathrm{mm}$, input speed is 
$n=2800 \mathrm{r} / \mathrm{min}$. For the simulation, the mechanical lubricating oil grade 22 \# is used and its main parameters are: $\alpha=2.1 \times 10^{-8} \mathrm{~m}^{2} / \mathrm{N}$ and $\eta_{0}=20 \times 10^{-3} \mathrm{~Pa} \cdot \mathrm{s}$. Figure 3 gives the overall technical route diagram of the methodology. Figure 4 gives the minimum oil film thickness at different positions of the drive system. It shows

Table 1. Parameter values of the drive system

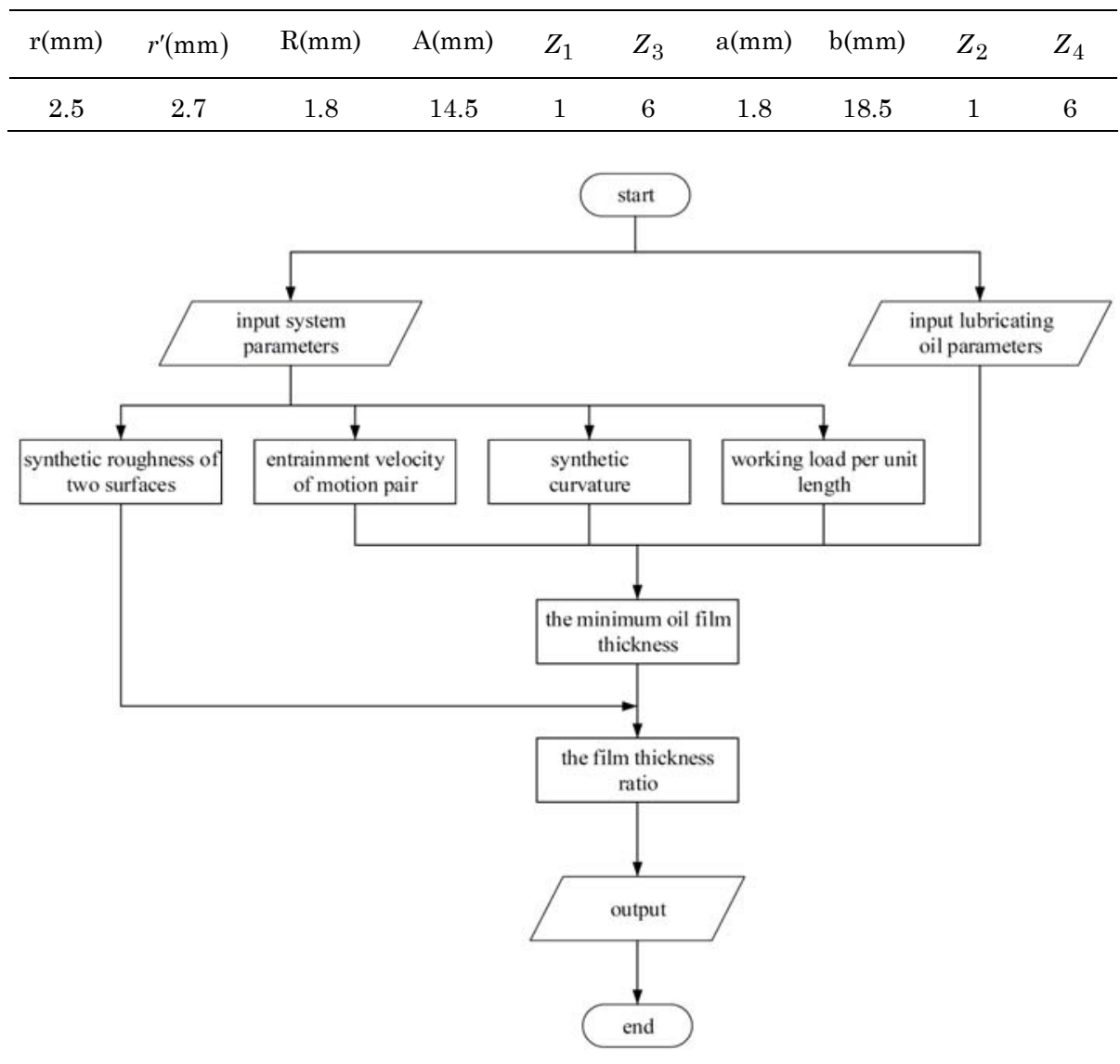

Figure 3. Overall technical route diagram of the methodology. 


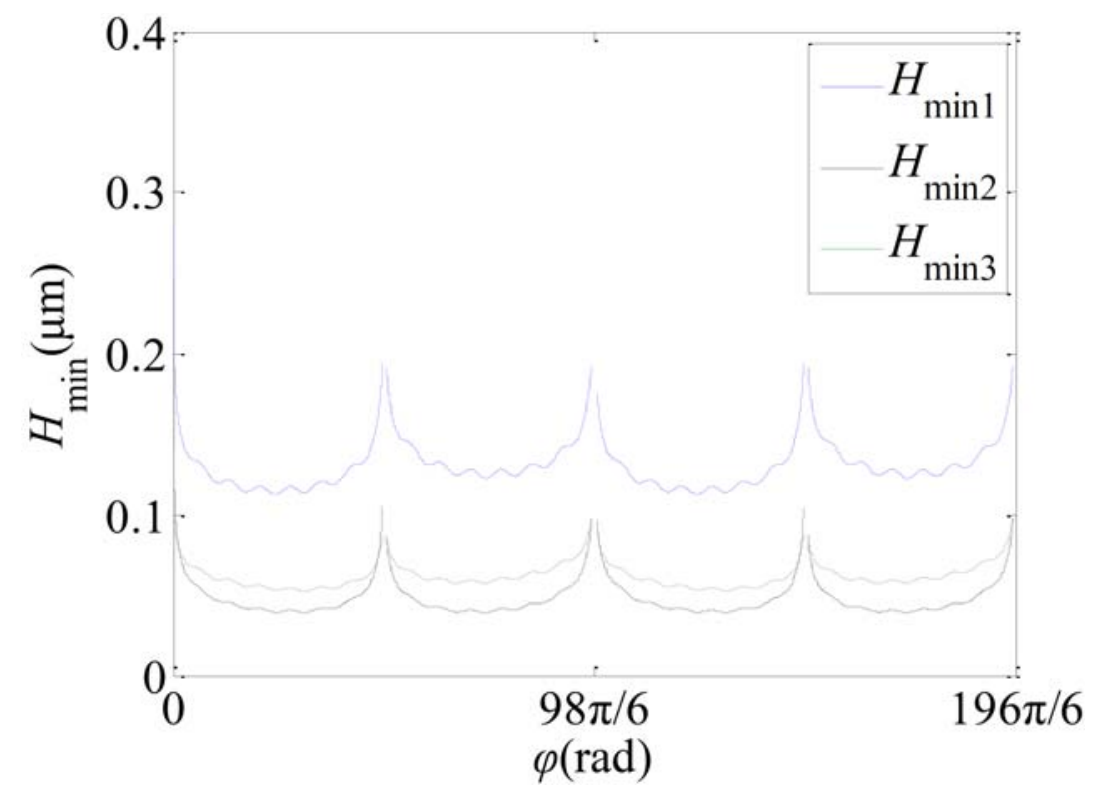

Figure 4. Minimum oil film thickness at different positions.

In the drive system, the minimum oil film thickness $H_{\min 1}$ between the movable tooth and the eccentric wheel at the meshing contact point is about $0.1146-0.1915 \mu \mathrm{m}$, and the minimum oil film thickness $H_{\min 2}$ between the movable tooth and the movable tooth carrier at the meshing contact point is about $0.039-0.1049 \mu \mathrm{m}$. The minimum oil film thickness $H_{\min 3}$ between the movable tooth and the central wheel at the contact point is about $0.0524-0.1000 \mu \mathrm{m}$.

Film thickness $H_{\min 1}$ is the largest among the three oil film thickness. Film thickness $H_{\min 2}$ is larger than $H_{\min 3}$. It is because entrainment velocities between the movable tooth and the eccentric wheel, the movable tooth carrier or central wheel has the following relation: $U_{1}>U_{2}>U_{3}$. 
The minimum oil film thickness is distributed periodically with rotation angle of the input shaft and the angle period is $49 \pi / 6$, which depends on the period of the entrainment velocity of the meshing pairs. Here, periodic peaks of the oil film thickness occur because forces between mesh elements are distributed periodically. The film thickness peaks correspond to small forces between mesh elements.

In order to effectively optimize the two-stage sinusoidal movable tooth drive, the effects of drive parameter and temperature on the minimum oil film thickness of drive system were studied (see Figure 5). In the study, it is found that the influence trend of the drive parameters on the minimum oil film thickness $H_{\min 1}, H_{\min 2}$, and $H_{\min 3}$ is the same. So, only the variation of $H_{\min 1}$ with drive parameters was given in Figure 5. Results show:

(1) As the eccentric distance $a$ of the eccentric wheel, the center distance $b$ between movable teeth and input shaft, and the radius $r$ of the movable teeth are increased, the minimum oil film thickness $H_{\min 1}$, $H_{\min 2}$, and $H_{\min 3}$ are increased.

(2) The eccentric distance $a$ of the eccentric wheel has the greatest influence on the minimum oil film thickness $H_{\min 1}$ between the movable teeth and the eccentric wheel. The effect of eccentric distance $a$ on the minimum oil film thickness $H_{\min 3}$ between movable teeth and central wheel is larger than $H_{\min 2}$ between movable teeth and movable tooth carrier.

(3) The center distance $b$ between the movable tooth and the input shaft has the greatest effect on the minimum oil film thickness $H_{\min 3}$ between the movable tooth and the central wheel. The effect of center distance $b$ on the minimum oil film thickness $H_{\min 2}$ between movable 
teeth and movable tooth frame is larger than $H_{\min 3}$ between movable teeth and central wheel.

(4) The radius $r$ of the movable tooth has the greatest influence on the minimum oil film thickness $H_{\min 1}$ between the movable teeth and the eccentric wheel. The effect of radius $r$ on the minimum oil film thickness $H_{\min 2}$ between movable teeth and movable tooth carrier is larger than $H_{\min 3}$ between movable teeth and central wheel.

(5) Among three drive parameters, eccentric distance $a$ of the eccentric wheel has the greatest influence on the minimum oil film thickness for the drive system. The effect of radius $r$ on the minimum oil film thickness of the drive system is larger than that of the center distance $b$.

(6) The minimum oil film thickness between meshing pairs decreases significantly with increasing temperature. The minimum oil film thickness at $55^{\circ} \mathrm{C}$ is only about $1 / 3$ at $25^{\circ} \mathrm{C}$ and $1 / 2$ at $40^{\circ} \mathrm{C}$. This is because the decrease in the viscosity of lubricating oil due to the rise in temperature. The viscosity of mechanical lubricating oil grade $22 \#$ at $25^{\circ} \mathrm{C}$, $40^{\circ} \mathrm{C}$, and $55^{\circ} \mathrm{C}$ is $43 \times 10^{-3} \mathrm{~Pa} \cdot \mathrm{s}, 20 \times 10^{-3} \mathrm{~Pa} \cdot \mathrm{s}$, and $7.5 \times 10^{-3} \mathrm{~Pa} \cdot \mathrm{s}$, respectively. 


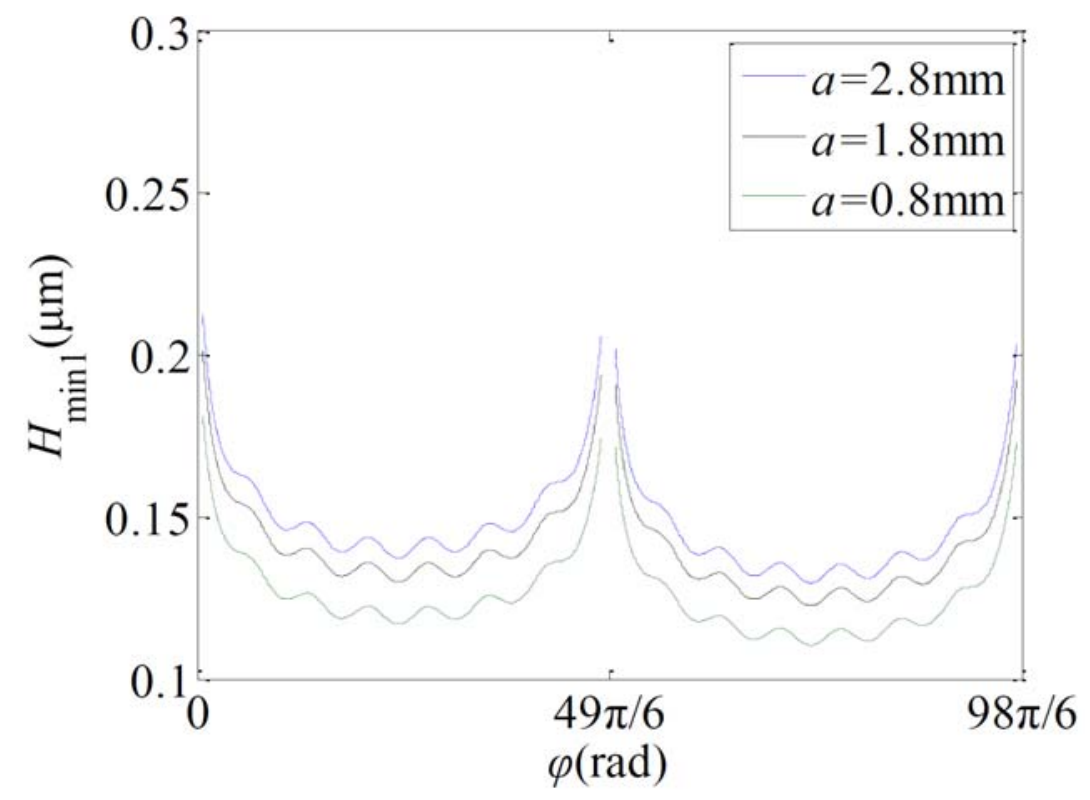

(a) $a$ changes

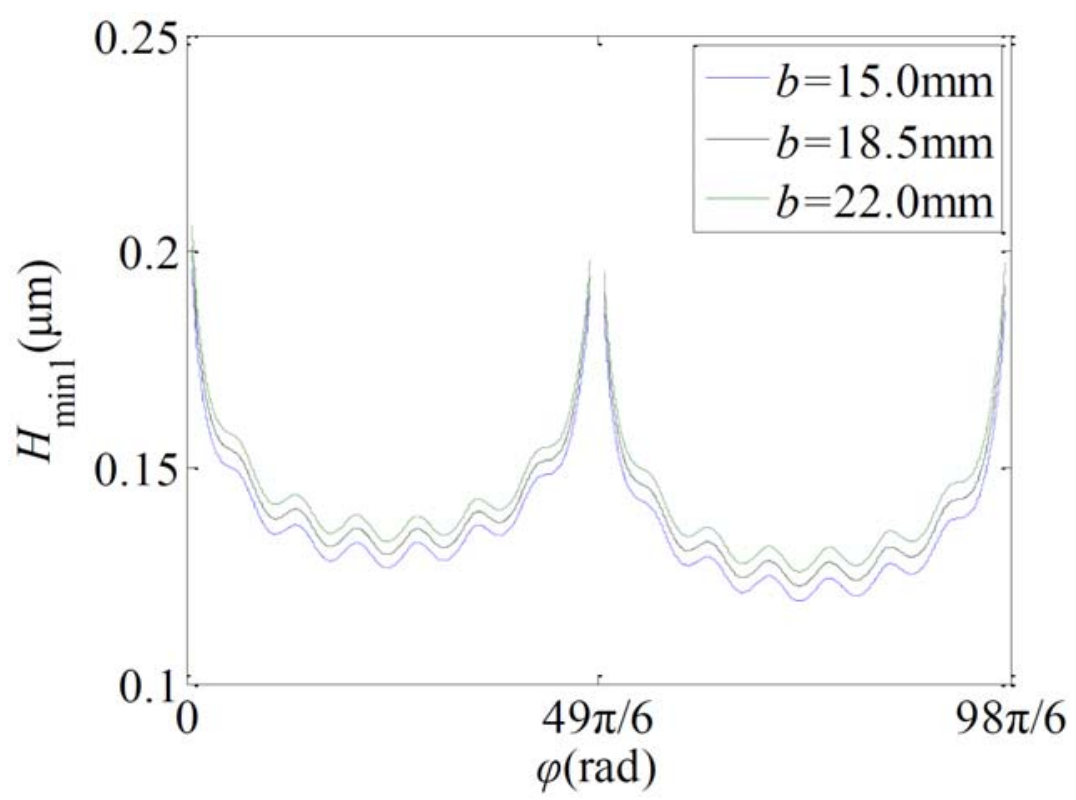

(b) $b$ changes 


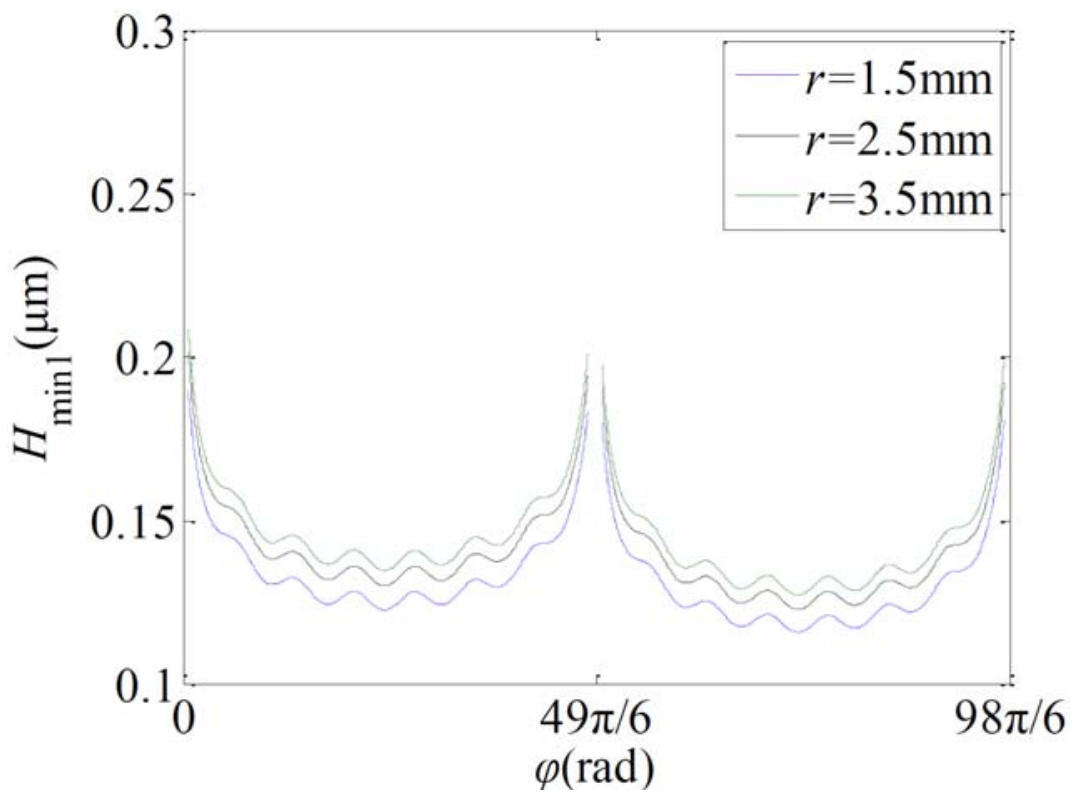

(c) $r$ changes

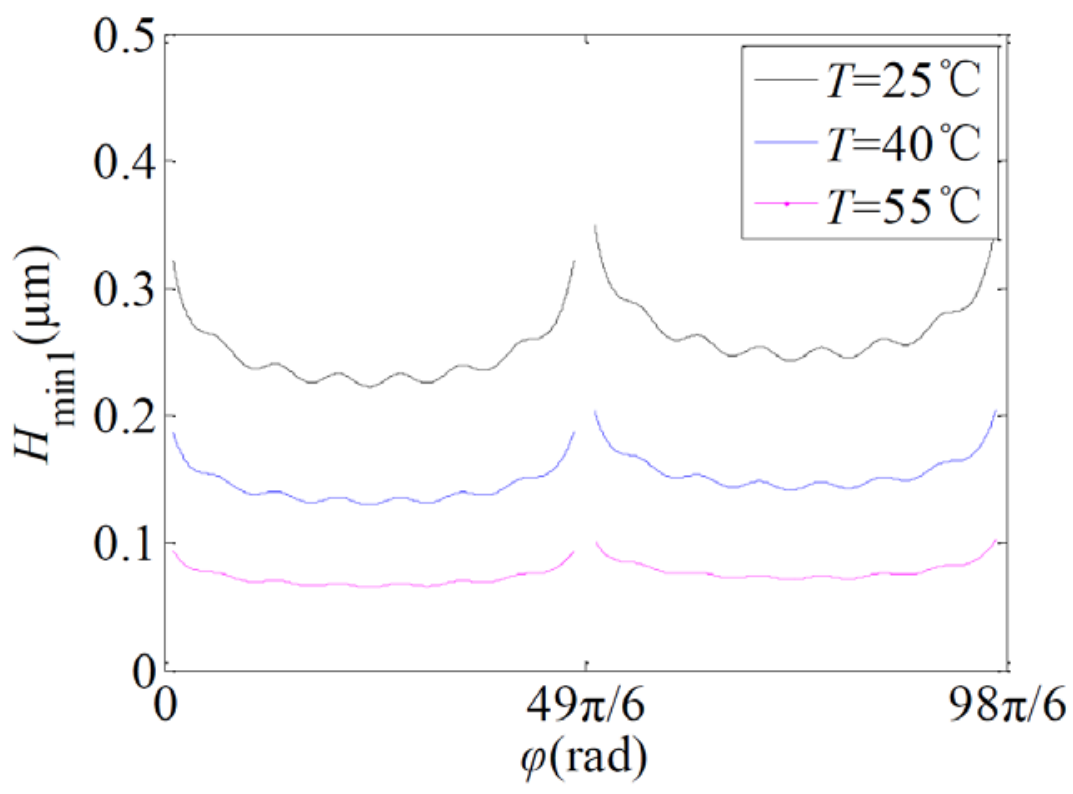

(d) $T$ changes

Figure 5. Effects of the drive parameters on $H_{\min 1}$. 
Let the root-mean-square values of two surface roughness be $\sigma_{1}$ and $\sigma_{2}$. The effective roughness of two surfaces can be calculated as $\sigma=\sqrt{\sigma_{1}{ }^{2}+\sigma_{2}{ }^{2}}$. Thus, film thickness ratio $\lambda$ can be determined as follows:

$$
\lambda=\frac{H_{\min }}{\sigma}=\frac{H_{\min }}{\sqrt{{\sigma_{1}}^{2}+{\sigma_{2}}^{2}}} .
$$

The surface roughness of the movable teeth and sinusoidal raceways can reach $0.20 \mu \mathrm{m}$ after grinding, and the effective roughness of the two surfaces is calculated to be about $0.40 \mu \mathrm{m}$. The above equation is used to calculate film thickness ratio $\lambda$ of the drive system (see Figure 6). Results show that film thickness ratio $\lambda$ of the drive system changes periodically with the rotation angle of the input shaft as well. However, the film thickness ratio $\lambda$ is smaller than 1 which shows that fluid lubrication state is not realized in the drive when the movable teeth and sinusoidal raceways of the main elements are grinded.

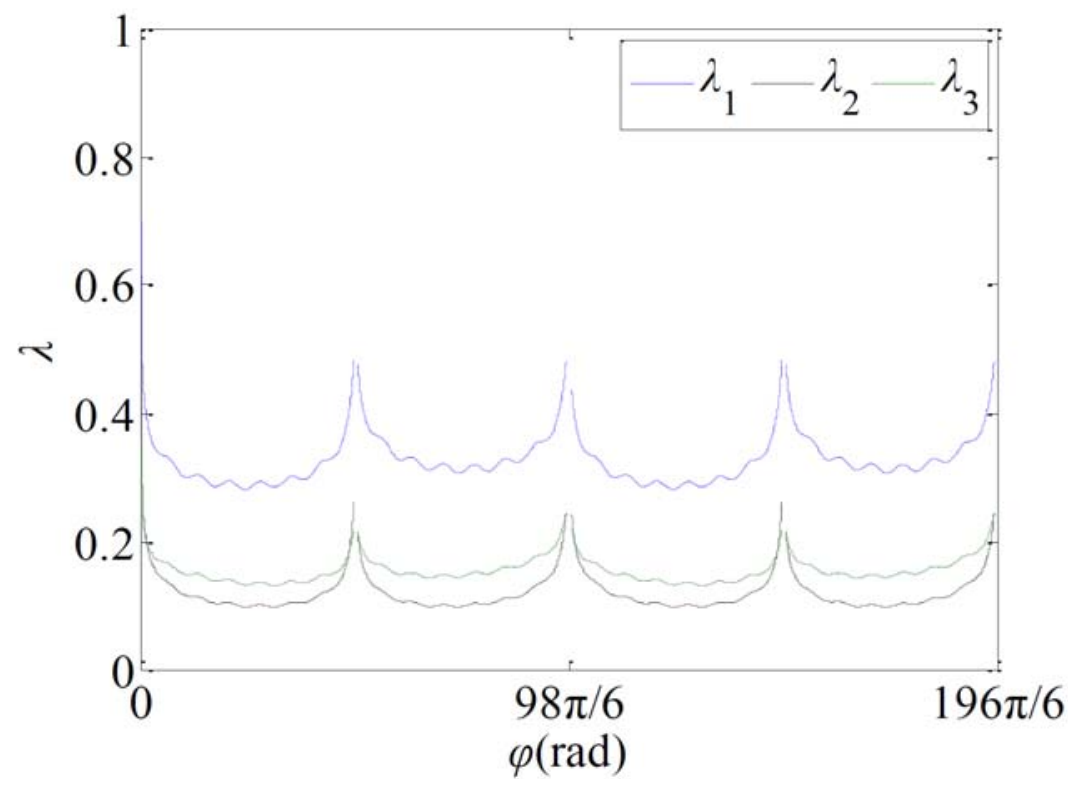

Figure 6. Film thickness ratio. 
In order to increase the film thickness ratio, the minimum oil film thickness can be increased or the effective roughness of the two surfaces can be reduced. The suction speed of the moving pair is proportional to the minimum oil film thickness, which can be increased by increasing the rotational speed. However, the calculation shows that the film thickness ratio can be greater than 1 only when the input shaft speed is greater than $80000 \mathrm{rpm}$, so it is not feasible.

In order to reduce the effective roughness of two surfaces, the surface accuracy of movable teeth and sinusoidal raceways should be improved. The surface roughness obtained by different machining methods is obtained by mechanical design handbook and shown in Table 2 .

Table 2. Surface roughness achieved by different methods

\begin{tabular}{cc}
\hline Process methods & roughness $(\mu \mathrm{m})$ \\
\hline Polish & $\mathrm{Ra}=0.63-0.01$ \\
Fine finish & $\mathrm{Ra}=0.2-0.012$ \\
Fine polish & $\mathrm{Ra} \leq 0.01$ \\
\hline
\end{tabular}

By calculation and analysis, it can be found that after the surface of movable teeth and sinusoidal raceways is treated by ultra-finishing, the film thickness ratio is greater than 1 , which can reach partial or full film elastic hydrodynamic lubrication state.

\section{Conclusion}

(1) The eccentric distance of eccentric wheel, the center distance between movable tooth and eccentric wheel, and the radius of movable tooth have important influence on the minimum oil film thickness in the two stage sinusoidal movable tooth drive. The minimum oil film thickness can be increased effectively by increasing the eccentric distance, the center distance and movable tooth radius. The increase of eccentricity decreases the working load per unit length, which is 
beneficial to the thickness of oil film. The increase of the center distance between the movable tooth and the eccentric wheel leads to the increase of the entrainment velocity and the decrease of the working load per unit length, both of which are beneficial to the thickness of the oil film. The increase of the radius of the movable teeth leads to the decrease of the working load per unit length and the increase of the curvature radius, both of which are beneficial to the oil film.

(2) The lubrication state between the movable tooth carrier and the movable tooth is the worst in the two-stage sinusoidal movable tooth drive. It should be taken as characteristic points of lubrication design. In order to ensure that the two-stage sinusoidal movable tooth drive is in an ideal elastic hydrodynamic state, the machining accuracy of the meshing pair surface can be reasonably selected and determined according to the minimum oil film thickness.

\section{Acknowledgement}

This project is supported by the Hebei Province Natural Science Foundation in China (No.E2017203021) and National key R \& D Program of China (No.2018YFB1304803).

\section{Nomenclature}

$F_{j i}:$ Force on the movable tooth from eccentric wheel;

$F_{s i}:$ Force on the movable tooth from the movable tooth frame;

$F_{g i}:$ Force on the movable tooth from central wheel;

$F_{j}$ : The maximum value of the force $F_{j i}$;

$F_{n i}$ : Normal force per unit length applied to movable tooth;

$E^{\prime}$ : Effective elastic modulus; 
$H_{\text {min }}$ : The minimum oil film thickness;

$H_{\min 1}$ : Oil film thickness between the movable tooth and eccentric wheel;

$H_{\min 2}:$ Oil film thickness between the movable tooth and movable tooth carrier;

$H_{\text {min3 }}$ : Oil film thickness between the movable tooth and central wheel;

$i_{2}:$ Speed ratio of the second stage drive;

$k_{v}$ : Induced normal curvature between meshing pairs;

$k$ : Curvature of the movable tooth;

$k^{\prime}:$ Curvature of the tooth slot;

$l:$ Length of the contact line;

$r:$ Radius of the movable tooth;

$r^{\prime}:$ Radius of the tooth slot;

$R_{S}:$ Effective curvature radius;

$R_{p}:$ Radius of the eccentric wheel;

$R_{s}$ : Effective radius of curvature between meshing pairs;

$S_{c}$ : Theoretical tooth profile of central wheel;

$t:$ The time;

$T$ : The temperature;

$T_{n 1}:$ Drive torque;

$U:$ Entrainment velocity; 
$U_{1}$ : Entrainment velocity between the movable tooth and eccentric wheel;

$U_{2}$ : Entrainment velocity between the movable tooth and movable tooth carrier;

$V_{m}$ : Motion velocity of the movable tooth;

$V_{H}$ : Motion velocity of the eccentric wheel;

$V_{K}:$ Motion velocity of the central wheel;

$x$ and $y$ : Coordinates of the tooth slot on eccentric wheel;

$W$ : Load per unit length;

$W_{1}$ : Load per unit length between the movable tooth and eccentric wheel;

$W_{2}$ : Load per unit length between the movable tooth and movable tooth frame;

$W_{3}$ : Load per unit length between the movable tooth and central wheel;

$Z_{2}$ : Period number of tooth slot on eccentric wheel;

$Z_{4}$ : Period number of the tooth slot on central wheel;

$Z_{t}$ : Coefficient related to parameters and position of the movable tooth;

$\alpha$ : Angle between force $F_{g i}$ and axis $y$;

$\alpha_{n}$ : Pressure viscosity coefficient;

$\gamma$ : Angle between force $F_{j i}$ and line of centers;

$\varepsilon$ : The maximum elastic deformation of the movable tooth; 
$\varepsilon_{i}$ : Elastic deformation of the movable tooth at any position;

$\eta_{0}:$ Dynamic viscosity of lubricant under normal pressure;

$\theta$ : Contacting half angle of the movable tooth;

$\lambda:$ The wave coefficient;

$\lambda_{n}:$ Film thickness ratio;

$\sigma:$ Effective roughness of two surfaces;

$\sigma_{1}$ and $\sigma_{2}$ : Root-mean-square values of two surface roughness in mesh;

$\varphi_{2}:$ Rotation angle of the eccentric wheel;

$\varphi_{4}$ : Rotation angle of the movable tooth carrier;

$\omega_{2}$ : Angular velocity of the guide frame (eccentric wheel);

$\omega_{3}$ : Angular velocity of the movable tooth carrier;

$\omega_{H}:$ Angular velocity of the center wheel;

$\omega_{m}$ : Angular velocity of the movable tooth around its axis.

\section{References}

[1] H. Terada, H. Makino and K. Imase, Fundamental analysis of cycloid ball reducer (1 $1^{\text {st }}$ Report) Motion Principle, Journal of the Japan Society for Precision Engineering 54(11) (1988), 2101-2106.

DOI: https://doi.org/10.2493/jjspe.54.2101

[2] H. Terada, H. Makino and K. Imase, Fundamental analysis of cycloid ball reducer ( $2^{\text {nd }}$ Report) radius of curvature and pressure angle, Journal of the Japan Society for Precision Engineering 56(4) (1990), 751-756.

DOI: https://doi.org/10.2493/jjspe.56.751

[3] S. Keith, Subtractive and Additive Differential Gear Reduction System: US, US4338830A[P], 1982. 
[4] I. Kenji, Ball-Rolling Type Torque Transmission Device: US, US5683323A[P].11-4, 1997.

[5] H. Terada and K. Imase, Fundamental analysis of a cycloid ball reducer (5 $5^{\text {th }}$ Report)development of a two stage type reduction mechanism, Journal of the Japan Society for Precision Engineering 75(12) (2009), 1418-1422.

DOI: https://doi.org/10.2493/jjspe.75.1418

[6] H. Terada, The development of gearless reducers with rolling balls, Journal of Mechanical Science and Technology 24(1) (2010), 189-195.

DOI: https://doi.org/10.1007/s12206-009-1155-0

[7] J. Han, An Electric Drum for Moving Teeth: China, CN203143543U[P].2013-08-21.

[8] X. Kong, Precision Cycloid Drive Gear Reducer: China, CN10235955[P].2012-02-22.

[9] Y. Wang and X. Chang, The synthesis and visualization on tooth profile of swing movable teeth transmission. Proceedings of the 2015 international conference on applied science and engineering innovation: AER-Advances in Engineering Research 12 (2015), 224-227.

[10] Y. Liang and L. Xu, Free vibration for an electromagnetic harmonic movable tooth drive system, Open Mechanical Engineering Journal 9(1) (2015), 15-21.

DOI: https://doi.org/10.2174/1874155X01509010015

[11] Y. Liang and L. Xu, Torque for an electromagnetic harmonic movable tooth drive system, Mechanism and Machine Theory 98 (2016), 190-198.

DOI: https://doi.org/10.1016/j.mechmachtheory.2015.12.014

[12] S. Liang, J. Zhang, L. Xu and Z. Liu, Dynamic model of swing movable teeth transmission system vibration, Journal of Vibration Engineering 2003(3) (2003), $285-289$.

[13] S. Liang and L. Li, Coupled thermo-elastic deformation of swing movable teeth transmission, Journal of Southwest Jiaotong University 3 (2017), 607-611.

[14] C. Li, J. Xing, J. Fang and Z. Zhao, Numerical analysis on chaotic vibration of drive system for a movable tooth piezoelectric motor, Shock and Vibration (2017); Article ID 3216010, 17 pages.

DOI: https://doi.org/10.1155/2017/3216010

[15] L. Xu, W. Song and R. Li, Two-step sine movable tooth drive, Advances in Mechanical Engineering 9(6) (2017), 1-9.

DOI: https://doi.org/10.1177/1687814017712414

[16] L. Xu and W. Wang, Quasi-static analysis of forces and stress for a novel two-step movable tooth drive. Mechanics Based Design of Structures and Machines 46(3) (2018), 285-295.

DOI: https://doi.org/10.1080/15397734.2017.1338961 
[17] M. Hammami, R. Martins, M. S. Abbes, M. Haddar and J. Seabra, Axle gear oils: Tribological characterization under full film lubrication, Tribology International 106 (2017), 109-122.

DOI: https://doi.org/10.1016/j.triboint.2016.05.051

[18] L. Xu and C. Yang, Elasto-hydrodynamic lubrication calculation and measure for crossed helical gears, Proceedings of the Institution of Mechanical Engineers, Part J: Journal of Engineering Tribology 220(6) (2006), 499-505.

DOI: https://doi.org/10.1243/13506501JET153

[19] D. Dowson and G. R. Higginson, Elasto-hydrodynamic Lubrication, Pergamon Press, Oxford, 1977. 